Revista Brasil. Bot., V.25, n.2, p.183-194, jun. 2002

\title{
Fenologia da copaíba (Copaifera langsdorffii Desf. - Leguminosae, Caesalpinioideae) em uma floresta semidecídua no sudeste do Brasil ${ }^{1}$
}

\author{
FERNANDO PEDRONI ${ }^{2,5}$, MARYLAND SANCHEZ ${ }^{3}$ e FLAVIO A.M. SANTOS ${ }^{4}$
}

(recebido: 30 de outubro de 2001; aceito: 01 de março de 2002)

\begin{abstract}
Phenology of copaíba (Copaifera langsdorffii Desf. - Leguminosae, Caesalpinioideae) in a semideciduous forest, southeastern Brazil). Plant phenology is concerned with the timing of recurring events, and is poorly known for tropical forest species, although this ecosystem has the greatest diversity of phenological patterns. A phenological study was carried out from February/1991 to April/1993. Thirty six trees were systematically observed biweekly for changes on leaf fall, leaf flushing, flowering and fruiting. Leaf fall and flushing were synchronized phases. These events always occurred at the end of dry season (leaf fall) and start of rainy season (leaf flushing) being clearly related with precipitation. The flowering occurred in the rainy season, and fruiting during the dry season. Fruiting did not show an annual cycle, but years which massive fruiting being followed by non productive years. Other non climatic factors, like seed dispersers and seed predators were also likely to affect fruiting.

RESUMO - (Fenologia da copaíba (Copaifera langsdorffii Desf. - Leguminosae, Caesalpinioideae) em uma floresta semidecídua no Sudeste do Brasil). A fenologia da emissão foliar, floração e frutificação para espécies de florestas tropicais é pouco conhecida, embora estes ecossistemas apresentem grande diversidade de padrões fenológicos. Foi estudada, de fevereiro de 1991 a abril de 1993, a fenologia da copaíba (Copaifera langsdorffii Desf.) na Reserva de Santa Genebra. Foram feitas observações em 36 indivíduos e analisadas queda de folhas, brotamento, floração e frutificação. A queda de folhas e o brotamento foram as fenofases mais sincronizadas dentro da população. Estes eventos ocorreram no final da estação seca (queda de folhas) e início da estação chuvosa (brotamento) e foram relacionados principalmente com a precipitação. A floração ocorreu na estação chuvosa e a frutificação na estação seca. A frutificação ocorreu em ciclos supra-anuais, com anos de produção intensa seguidos de anos sem frutificação. Além dos fatores climáticos, as interações com dispersores e predadores de sementes também parecem influenciar o padrão de frutificação observado em Copaifera langsdorffii.
\end{abstract}

Key words - Seasonality, leaf fall, leaf flushing, flowering, fruiting

\section{Introdução}

A fenologia das plantas pode ser influenciada por fatores próximos e fatores finais. Fatores próximos incluem precipitação (Opler et al. 1976), estresse hídrico (Borchert 1980, Reich \& Borchert 1984), irradiação (Wright \& van Schaik 1994) e fotoperíodo (Rivera \& Borchert 2001), enquanto fatores finais incluem a reprodução cruzada entre indivíduos e abundância de polinizadores (Augspurger 1996), dispersores (Snow 1965) e predadores de sementes (Janzen 1971).

Nas florestas tropicais, enquanto relações marcantes entre períodos de estiagem e queda de folhas têm

1. Parte da dissertação de mestrado do $1^{\circ}$ autor, junto ao Programa de Pós-graduação em Biologia Vegetal, Unicamp.

2. Universidade Federal de Uberlândia, Instituto de Biologia, Caixa Postal 593, 38400-902 Uberlândia, MG, Brasil.

3. Universidade de Brasília, Departamento de Engenharia Florestal.

4. Universidade Estadual de Campinas, Instituto de Biologia, Departamento de Botânica, Caixa Postal 6109, 13083-970 Campinas, SP, Brasil.

5._Autor para correspondência: fpedroni@umuarama.ufu.br sido encontradas por diferentes autores (Morellato et al. 1989, Justiano \& Fredericksen 2000), o sincronismo do brotamento tem sido relacionado a vários fatores como: mudanças na disponibilidade de água e luz (Lieberman \& Lieberman 1984, Borchert 1994, Reich 1995) e também com as flutuações nas populações de herbívoros (Aide 1993, Angulo-Sandoval \& Aide 2000).

Eventos fenológicos reprodutivos sazonais e sincronizados podem representar vantagens adaptativas para muitas espécies tropicais. Algumas plantas iniciam a produção de flores estimuladas pela variação sazonal na irradiação solar (Wright \& van Schaik 1994) e abrem seus frutos no final da estação seca e início da chuvosa para reduzir a mortalidade das plântulas (Frankie et al. 1974, van Schaik et al. 1993). Fatores fisiológicos também podem influenciar a sazonalidade da floração e frutificação (Primack 1985a). O ciclo reprodutivo das plantas pode ser afetado pela quantidade de nutrientes disponíveis no solo (Zagt 1997) e, muitas vezes, quando a deficiência de recursos torna-se acentuada em função de frutificações anteriores, um padrão supra-anual de frutificação pode ser observado (Flowerdew \& Gardner 1978). Florescer sincronizadamente favorece a atração 
de polinizadores facilitando o fluxo de pólen e a reprodução cruzada (Marquis 1988). Da mesma maneira, a produção sincronizada de frutos pode saciar os predadores de sementes (Janzen 1971, Tapper 1992) e aumentar a atratividade de frugívoros necessários para a dispersão de sementes (Smythe 1970).

O tempo, duração e grau de sincronia das várias fases fenológicas têm importantes implicações sobre a quantidade e qualidade dos recursos disponíveis para os organismos consumidores (polinizadores, dispersores e predadores) e influencia a estrutura, funcionamento e regeneração das comunidades (Williams et al. 1999).

Nos trópicos, fatores climáticos e interações entre espécies estão freqüentemente associados com a sazonalidade dos eventos vegetativos e reprodutivos das plantas, tanto em nível de comunidade (e.g. Frankie et al. 1974, Morellato et al. 1989, 2000, Miranda 1995, Batalha et al. 1997, Batalha \& Mantovani 2001, Machado et al. 1997, WilliansLinera 1997, Justiniano \& Fredericksen 2000, Silberbauer-Gottsberger 2001), quanto específico (Dias \& Oliveira-Filho 1996, Felfili et al. 1999, Adler \& Kielpinski 2000, Almeida \& Alves 2000, Harrison et al. 2000). Segundo Newstrom et al. (1994), os padrões fenológicos de plantas tropicais podem ser diferentes dependendo do nível (comunidade, população ou indivíduo) em que são analisados. Estudos recentes têm destacado a importância de proceder as análises em níveis hierárquicos mais baixos para melhor entendimento dos padrões fenológicos (Sun et al. 1996).
A fenologia de Copaifera langsdorffii Desf., conhecida popularmente como copaíba, é pouco conhecida no Estado de São Paulo (Crestana \& Kageyama 1989) e, neste trabalho, foram avaliados os eventos fenológicos apresentados por esta espécie na Reserva de Santa Genebra e discutidas as suas relações com as variações climáticas e com os animais dispersores e predadores de sementes.

\section{Material e métodos}

O estudo foi realizado na Reserva Municipal de Santa Genebra (RSG), localizada no distrito de Barão Geraldo, região norte do Município de Campinas, SP (22 $49^{\prime} \mathrm{S}$; $\left.47^{\circ} 06^{\prime} \mathrm{W}\right)$.

Segundo a classificação de Köppen (1948), o clima da região é do tipo $\mathrm{CW} 2$. Apresenta uma precipitação média anual de 1.381,2 mm com uma estação quente e úmida (74\% da precipitação anual), com temperaturas médias mensais entre 20 e $25{ }^{\circ} \mathrm{C}$ (outubro-março) e uma estação fria e seca (26\% da precipitação anual), com temperaturas médias mensais entre 17 e $20^{\circ} \mathrm{C}$ (abril-setembro). Nos anos de 1990 a 1992, foi observada queda na pluviosidade do mês de fevereiro, durante a estação chuvosa, sendo que esta redução foi mais crítica na estação chuvosa de 1991/92 (figura 1). Os dados de temperatura e pluviosidade foram obtidos na Estação Meteorológica do Instituto Agronômico de Campinas (IAC), Fazenda Santa Eliza, a $669 \mathrm{~m}$ de altitude, distante cerca de $4 \mathrm{~km}$ em linha reta da Reserva.

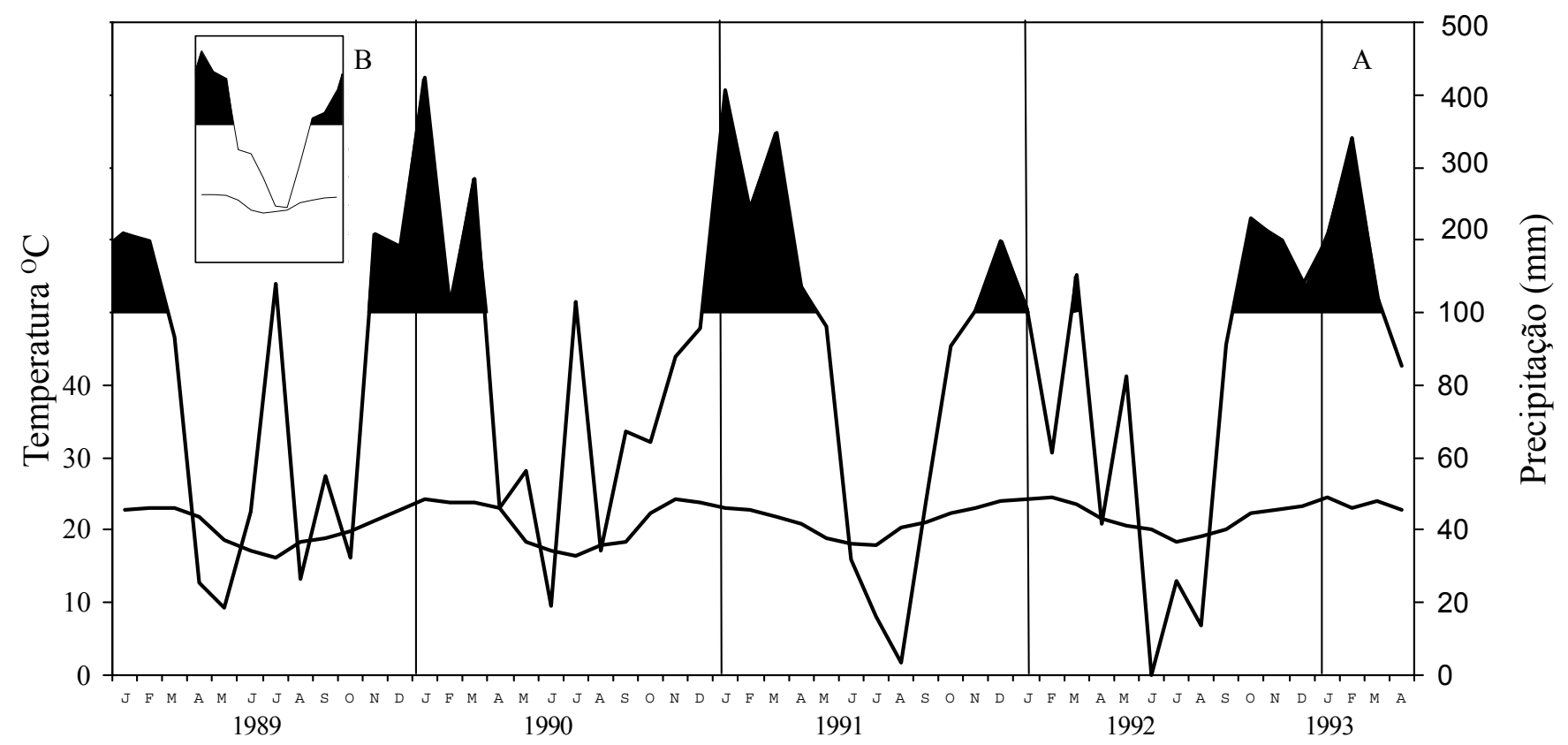

Figura 1. Diagrama climatológico para os anos de 1989 a 1993 (A) e normais climatológias da região de Campinas (B). Dados registrados pela estação meteorológica do Instituto Agronômico de Campinas (IAC). 
O solo da área pertence à unidade Barão Geraldo, cuja classificação é latossolo roxo distrófico (Oliveira 1980). A topografia da região é formada por colinas suavizadas, com relevo ondulado, numa altitude média de $700 \mathrm{~m}$, ocorrendo afloramentos d'água nas áreas mais baixas que permanecem alagadas o ano todo.

A vegetação da RSG pode ser classificada como Floresta Estacional Semidecidual (Veloso et al. 1991). Essa floresta foi fragmentada durante as décadas de 50 e 70 restando, atualmente, cerca de 250 ha circundados por plantações de milho, soja, algodão, mandioca e cana-de-açúcar e, mais recentemente, por habitações humanas (Leitão Filho 1995).

Três subtipos de vegetação são encontrados na Reserva: a floresta semidecídua propriamente dita, a floresta úmida ou de brejo e a vegetação secundária ou de beira de mata. A floresta semidecídua cobre a maior parte da reserva, apresenta diferentes graus de perturbação e é caracterizada por um dossel quase contínuo a cerca de 15 a $20 \mathrm{~m}$ de altura e árvores emergentes com até $30 \mathrm{~m}$. Sob o dossel forma-se um estrato composto por arbustos e arvoretas com até $8 \mathrm{~m}$ de altura e um estrato herbáceo pouco desenvolvido (Leitão Filho 1995).

$\mathrm{Na}$ RSG, Copaifera langsdorffii atinge mais de $20 \mathrm{~m}$ de altura, ocorrendo principalmente nas áreas de floresta semidecídua e bordas da mata. As folhas são alternas, compostas paripinadas, com 3 a 5 jugos, folíolos opostos ou alternos, glabros e as folhas novas são avermelhadas. As flores são brancas, pequenas, hermafroditas, reunidas em panículas terminais. Os frutos são do tipo folículo com uma semente preta envolvida por um arilo de cor laranja. Este conjunto de características morfológicas do fruto indicam uma planta dispersa por animais, principalmente aves. O material botânico testemunho foi depositado no Herbário da Universidade Estadual de Campinas (UEC) sob o número de registro 27.093.

Os dados utilizados neste trabalho foram obtidos de duas maneiras distintas. Nos anos de 1989 e 1990, foram obtidas informações fenológicas referentes à fase reprodutiva durante um estudo sobre frugivoria (Pedroni 1995). Nesta fase, foram instalados 32 coletores de $1 \mathrm{~m}^{2}$ sob a copa de oito indivíduos (4 coletores/indivíduo) para estimar a produtividade de frutos da população de Copaifera langsdorffii. Após este período, foram feitas observações sistemáticas em oito até 36 indivíduos. Os números amostrais foram diferentes em cada ano devido à inclusão de indivíduos ou perda causada pela queda das árvores. Os indivíduos escolhidos para observações fenológicas apresentaram entre 20 e $105 \mathrm{~cm}$ de DAP (diâmetro à altura do peito) e altura entre seis e $20 \mathrm{~m}$. Todos foram considerados adultos uma vez que o menor indivíduo observado frutificou durante o período de estudo.

Os indivíduos marcados foram observados quinzenalmente. As seguintes fenofases foram acompanhadas: 1) queda de folhas - anotada sempre que as folhas das árvores caiam com facilidade ao ventar, quando havia muitas folhas, da própria árvore, caídas sob a copa ou quando eram observadas falhas (espaços vazios) na copa ou galhos; 2) brotamento - início: a partir do aparecimento de pequenas folhas, brilhantes com coloração vermelha; fim: quando a maioria das folhas atingiam aproximadamente $3 / 4$ do tamanho de uma folha adulta e apresentavam coloração verde claro brilhante; 3) folhas novas - considerada a partir do final do brotamento até as folhas atingirem o tamanho e cor (verde escuro com pouco brilho) característicos das folhas maduras; 4) folhas maduras - folhas completamente desenvolvidas com coloração verde escura e pouco brilho; 5) floração subdividida em botões e flores abertas, esta última considerada a partir da observação da antese dos primeiros botões até a queda de todas as flores; 6) frutificação subdividida em frutos imaturos e frutos maduros, esta última inicia-se com a observação dos primeiros frutos abertos e dura até o final da dispersão das sementes.

Para relacionar os fatores abióticos (precipitação e temperatura do período de estudo e normais climatológicas de precipitação e temperatura) com as fenofases (brotamento, queda de folhas, floração e frutificação) foi utilizada a correlação de Spearman $\left(\mathrm{r}_{\mathrm{s}}\right)$ que é recomendada para dados que não apresentam distribuição normal (Zar 1999).

Para verificar a sincronia das diferentes fenofases na população foi utilizado o Índice de sincronia $(Z)$ adaptado de Augspurger (1983), que foi definido como:

$$
\mathrm{Z}=\sum_{\mathrm{I}=1}^{\mathrm{n}} \mathrm{X}_{\mathrm{i}} / \mathrm{n}
$$

onde, $\mathrm{X}_{\mathrm{i}}=$ sincronia do indivíduo $\mathrm{i}$ com seus coespecíficos

$\mathrm{n}=$ número de indivíduos na população

Calcula-se $X_{i}$ através da seguinte fórmula:

$$
\mathrm{X}_{\mathrm{i}}=(1 / \mathrm{n}-1) \cdot\left(1 / \mathrm{f}_{\mathrm{i}}\right) \cdot \sum_{\mathrm{i}=1}^{\mathrm{n}} \mathrm{e}_{j \neq \mathrm{i}}
$$

onde, $\mathrm{e}_{\mathrm{j}}=$ número de registros nos quais ambos os

indivíduos i e $\mathrm{j}$ estão na mesma fenofase, $\mathrm{j} \neq \mathrm{i}$

$\mathrm{f}_{\mathrm{i}}=$ número de registros nos quais o indivíduo i está na fenofase considerada

Quando $Z=1$ ocorre a sincronia perfeita, isto é, todos os indivíduos da população apresentam-se simultaneamente na mesma fase fenológica. Quando $Z=0$, não ocorre nenhuma sincronia entre os indivíduos da população.

\section{Resultados}

A fenologia em nível individual $(\mathrm{n}=36)$ variou pouco com relação à fase vegetativa (queda de folhas e brotamento) e amplamente para a fase reprodutiva (floração e frutificação), durante o período de fevereiro de 1991 a abril de 1993 (figura 2).

Queda de folhas e brotamento - Copaifera langsdorffii apresentou marcada sazonalidade na queda de folhas. Os indivíduos perderam grande proporção de folhas, mas em nenhum momento as copas ficaram completamente desfolhadas. Este evento ocorreu no final da 


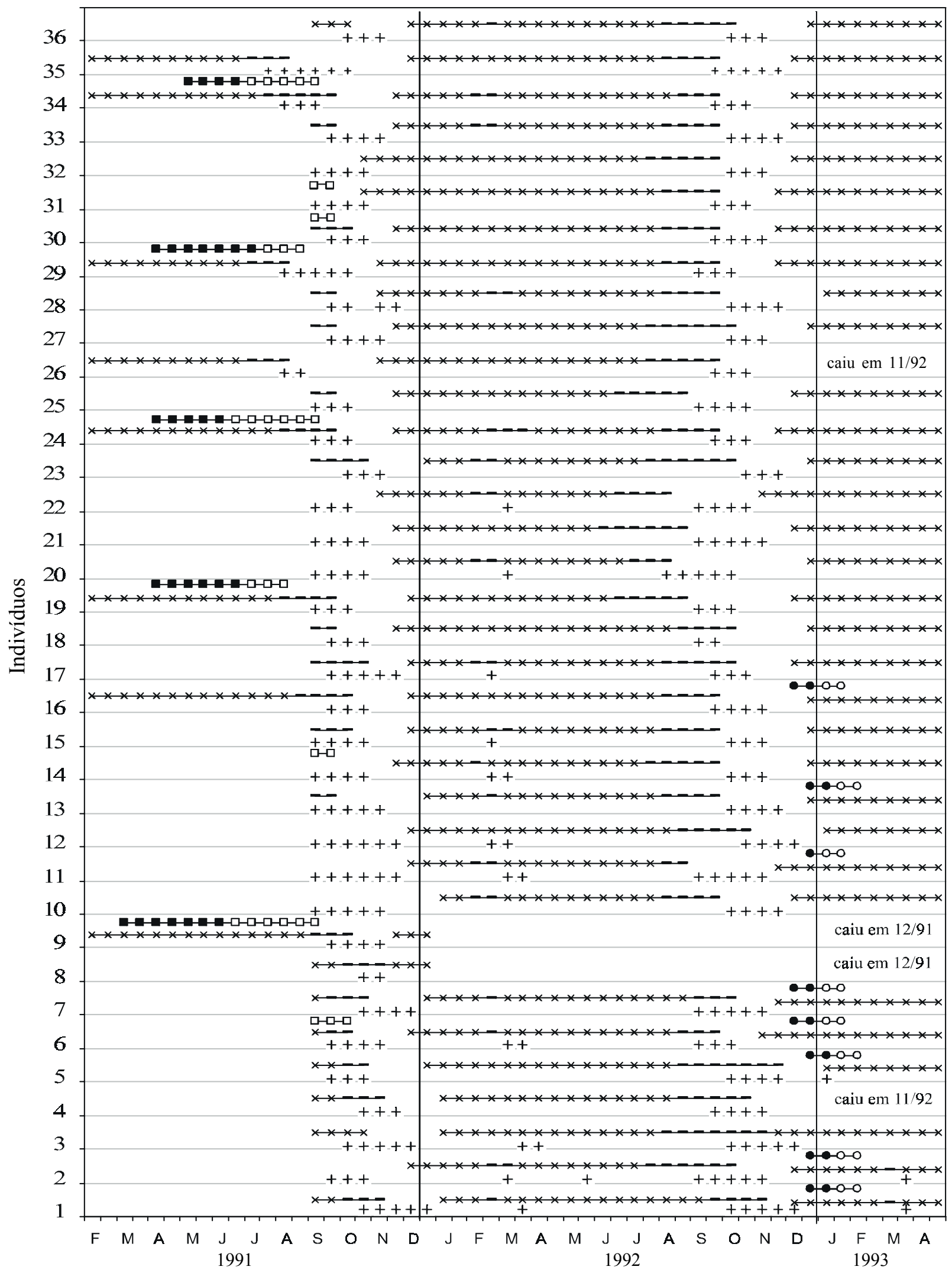

- Queda de folhas; + Brotamento; - - X-Folhas maduras; - -- Botões; -O-Flores abertas; - $\mathbf{-}$ - Frutos imaturos; - $\square$ - Frutos maduros.

Figura 2. Eventos fenológicos de cada indivíduo da população de Copaifera langsdorffii estudada na floresta semidecídua da RSG, Campinas (SP). 
estação seca (julho-agosto) que corresponde a época de maior estresse hídrico (figura 1). Esta fenofase foi correlacionada significativa e negativamente com a precipitação mensal total do período de observação $\left(\mathrm{r}_{\mathrm{s}}=-0,58 ; \mathrm{p}=0,01\right)$, com a precipitação $\left(\mathrm{r}_{\mathrm{s}}=-0,47\right.$; $\mathrm{p}=0,01)$ e temperatura médias $\left(\mathrm{r}_{\mathrm{s}}=-0,37 ; \mathrm{p}=0,03\right)$ das normais climatológicas. A maioria da população (entre 75 e $90 \%$ dos indivíduos) perdeu folhas simultaneamente em julho-agosto (figura 3) apresentando índice de sincronia $(Z)$ de $0,45 \mathrm{em} 1991$ e de $0,68 \mathrm{em}$ 1992. No mês de fevereiro de 1992, houve uma redução importante na precipitação em relação aos dados normais (figura 1). Neste mês, até início de março, ocorreu queda de folhas pouco pronunciada em $50 \%$ dos indivíduos o que se refletiu no baixo índice de sincronia apresentado pela população $(Z=0,19)$ neste período.

No final da estação seca e início da chuvosa (setembro-outubro), ocorreu o brotamento, facilmente observado devido ao aspecto avermelhado das copas neste período. $\mathrm{O}$ brotamento não foi significativamente correlacionado com os dados climatológicos considerados. A maioria da população (entre 80 e $90 \%$ do indivíduos) emitiu folhas novas em setembro (figura 3) apresentando índice de sincronia (Z) de 0,60 em 1991 e de 0,67 em 1992. Os indivíduos que perderam folhas no período de fevereiro-março de 1992 (figura 3) apresentaram um subseqüente brotamento que ocorreu de forma sincronizada $(Z=0,43)$.

Floração e frutificação - A floração foi observada apenas na estação chuvosa de 1992-93, principalmente em dezembro e janeiro e ocorreu somente em $25 \%$ da população (figura 3). Em alguns indivíduos esta fase fenológica durou até fevereiro (figura 2). No ano de 1991, ocorreu floração mas o acompanhamento fenológico sistemático ainda não havia sido iniciado. O período entre o aparecimento dos primeiros botões até a antese das últimas flores durou 50 dias. O tempo médio de floração por indivíduo foi de 35,25 dias $( \pm 3,03)$. Em diferentes indivíduos, grupos de botões se abriram em intervalos de uma semana até 15 dias. $\mathrm{O}$ tempo de duração das flores após a antese foi de apenas um dia. Não houve correlação significativa entre a floração e os parâmetros climáticos considerados. Em razão do pequeno número de indivíduos que floresceram no período, a população como um todo apresentou baixa sincronia $(Z=0,04)$, mas considerando-se apenas os indivíduos que floresceram ocorreu uma elevada sincronia $(Z=0,75)$.

Durante o período de 1989 a 1993, a ocorrência da frutificação variou anualmente e entre os indivíduos da população. Seis indivíduos frutificaram consecutivamente em 1990 e 1991, pelo menos cinco indivíduos frutificaram apenas uma vez durante o período de estudo, e 13 indivíduos não foram observados frutificando em nenhuma ocasião. Apenas em um indivíduo foram observados três eventos de frutificação (tabela 1). Em 1989 e 1992, nenhum dos indivíduos observados frutificou. No ano de 1990, 68\% dos indivíduos observados frutificaram (tabela 1). A produtividade média foi de $8.368 \pm 9.738$ frutos/ indivíduo. $\mathrm{O}$ indivíduo mais produtivo rendeu mais de 30.000 frutos e, no ano seguinte, não produziu nem 10\% da safra de 1990. O menos produtivo rendeu apenas 298 frutos em 1990 e não frutificou nos anos seguintes. Em 1991, apenas 25\% das plantas acompanhadas (tabela 1) produziram pequena quantidade de frutos (produtividade média 3.233 \pm 401). No ano de 1993, embora $25 \%$ população tenha sido observada frutificando, a produtividade foi muito baixa, não sendo possível estimá-la com uso de coletores. Os frutos desenvolveram-se durante a estação seca (abril-setembro), ocorrendo a deiscência e dispersão das sementes no final do período de estiagem e início do chuvoso. No início da estação chuvosa (outubro), praticamente todos os frutos já não apresentavam mais sementes e algumas valvas permaneceram presas aos indivíduos até novembro. O tempo médio de desenvolvimento dos frutos até a dispersão das sementes foi de 129,32 dias $( \pm 15,43)$. Foi possível encontrar frutos maduros em cada indivíduo observado por um período médio de 45 dias $( \pm 7,54)$. Não houve correlação significativa entre a frutificação e os parâmetros climáticos considerados. Dentre os indivíduos que frutificaram foi observada uma elevada sincronia $(Z=0,88)$, enquanto para a população a sincronia foi menor $(Z=0,28)$.

\section{Discussão}

O ritmo fenológico apresentado por Copaifera langsdorffii na RSG caracterizou-se pela ocorrência seqüencial das diferentes fenofases: a queda de folhas ocorreu principalmente no final da estação seca (julhoagosto), o brotamento no final da estação seca e início da estação chuvosa (setembro-outubro), a floração, em meados da estação chuvosa (dezembro-fevereiro), o desenvolvimento dos frutos ocorreu durante a estação seca (abril-setembro) e a dispersão, ao final desta estação (agosto-setembro). Este padrão foi semelhante ao encontrado por Dias \& Oliveira-Filho (1996) em uma floresta estacional semidecídua montana em Lavras (MG). Por outro lado, Batalha et al. (1997) observaram 

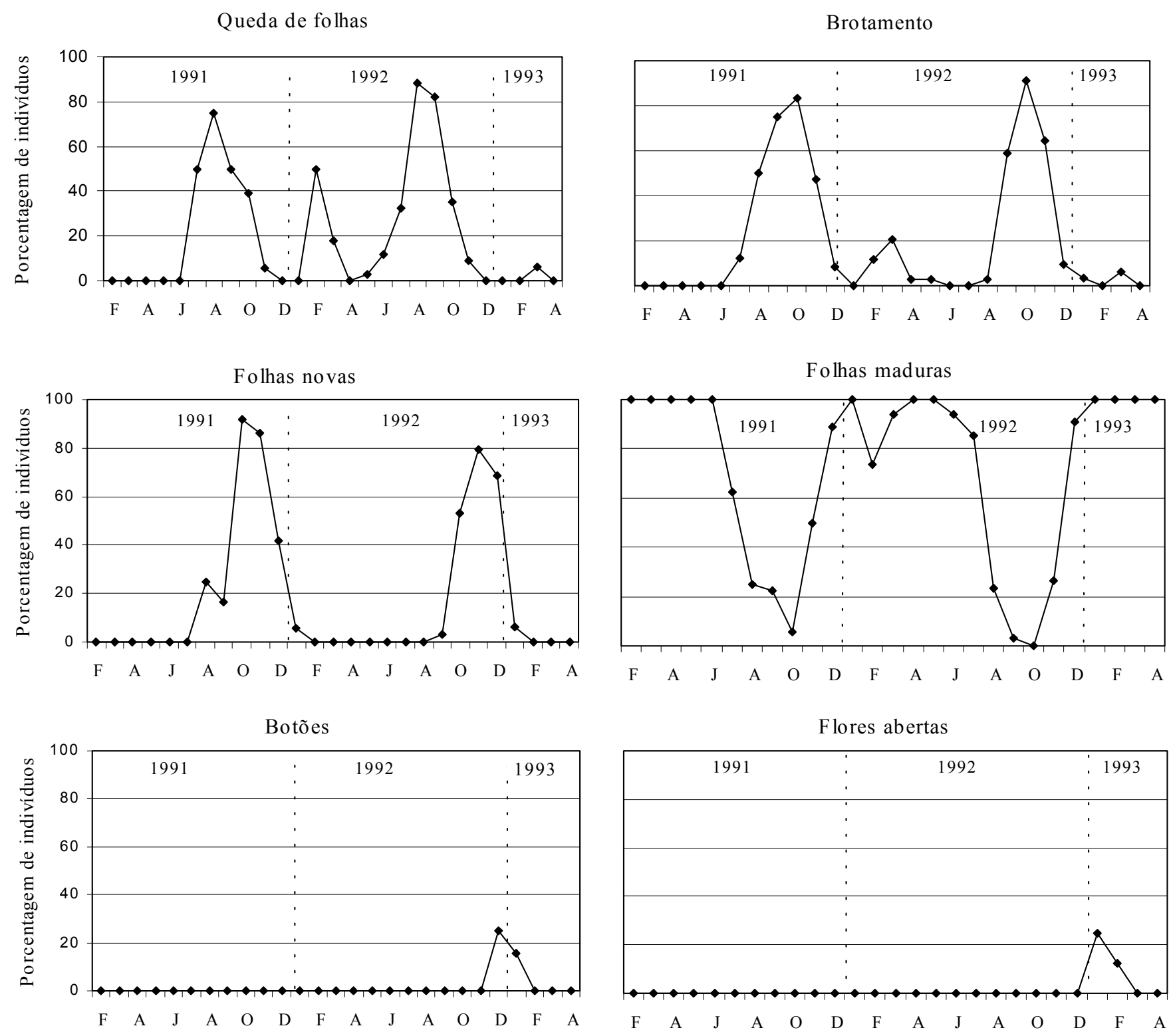

Flores abertas

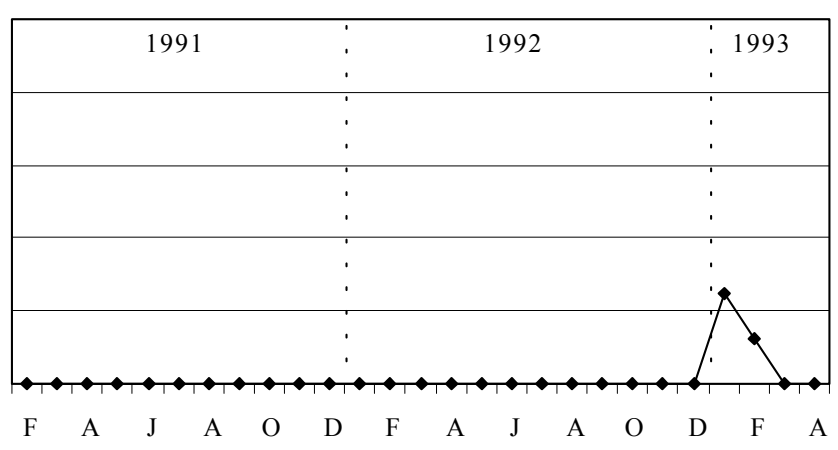

Frutos imaturos

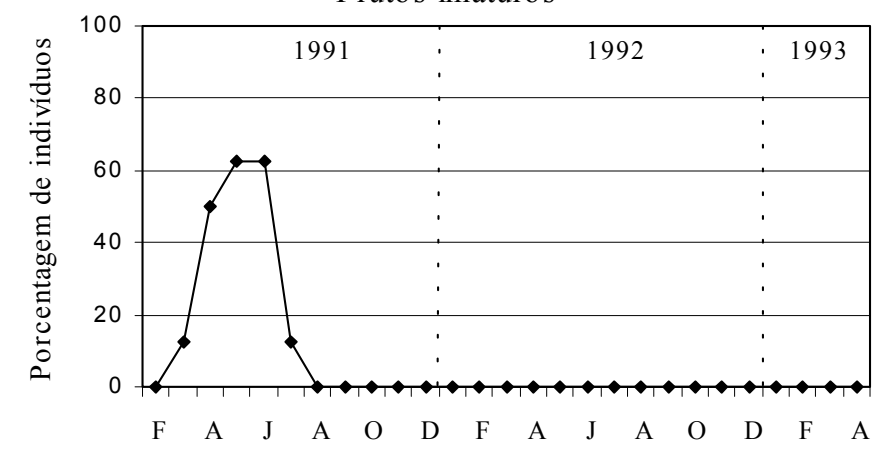

Frutos maduros

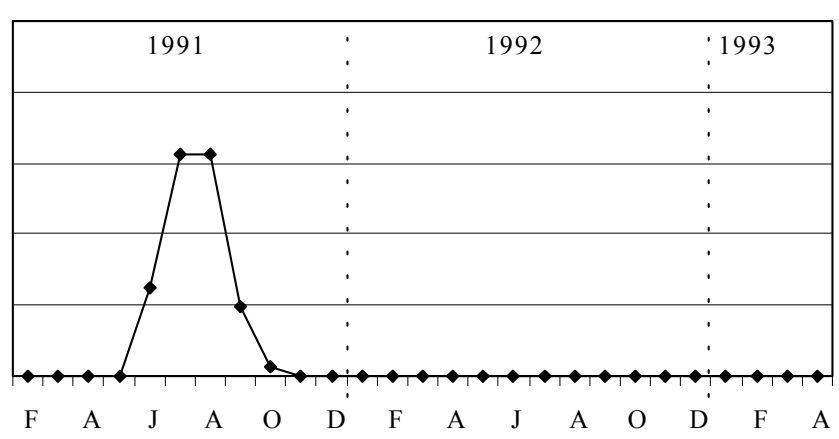

Figura 3. Fenofases apresentadas por Copaifera langsdorffii de fevereiro/1991 a abril/1993, na floresta semidecídua da RSG, Campinas (SP). 
Tabela 1. Eventos reprodutivos dos indivíduos de Copaifera langsdorffii durante os anos de estudo (Informações complementares para 1989/1990 foram fornecidas por L. P. Morellato). (Fr): ocorrência de frutificação; (-): não ocorrência de frutificação; (espaços em branco): indicam ausência de informação; $(+)$ : indivíduos que caíram ou morreram durante o período de estudo.

\begin{tabular}{|c|c|c|c|c|c|}
\hline IND & 1989 & 1990 & 1991 & 1992 & 1993 \\
\hline 1 & & $\mathrm{Fr}$ & - & - & $\mathrm{Fr}$ \\
\hline 2 & & & - & - & $\mathrm{Fr}$ \\
\hline 3 & & & - & - & - \\
\hline 4 & & $\mathrm{Fr}$ & - & + & + \\
\hline 5 & & & - & - & $\mathrm{Fr}$ \\
\hline 6 & & $\mathrm{Fr}$ & $\mathrm{Fr}$ & - & $\mathrm{Fr}$ \\
\hline 7 & & & - & - & $\mathrm{Fr}$ \\
\hline 8 & & & - & + & + \\
\hline 9 & & $\mathrm{Fr}$ & $\mathrm{Fr}$ & + & + \\
\hline 10 & & - & - & - & - \\
\hline 11 & & & - & - & $\mathrm{Fr}$ \\
\hline 12 & & & - & - & - \\
\hline 13 & & & - & - & $\mathrm{Fr}$ \\
\hline 14 & & & $\mathrm{Fr}$ & - & - \\
\hline 15 & & & - & - & - \\
\hline 16 & & $\mathrm{Fr}$ & - & - & $\mathrm{Fr}$ \\
\hline 17 & & - & - & - & - \\
\hline 18 & & - & - & - & - \\
\hline 19 & - & $\mathrm{Fr}$ & $\mathrm{Fr}$ & - & - \\
\hline 20 & - & $\mathrm{Fr}$ & - & - & - \\
\hline 21 & & $\mathrm{Fr}$ & - & - & - \\
\hline 22 & & & - & - & - \\
\hline 23 & - & $\mathrm{Fr}$ & - & - & - \\
\hline 24 & - & $\mathrm{Fr}$ & $\mathrm{Fr}$ & - & - \\
\hline 25 & - & $\mathrm{Fr}$ & - & - & - \\
\hline 26 & & - & - & - & + \\
\hline 27 & & - & - & - & - \\
\hline 28 & & & - & - & - \\
\hline 29 & & $\mathrm{Fr}$ & $\mathrm{Fr}$ & - & - \\
\hline 30 & - & $\mathrm{Fr}$ & $\mathrm{Fr}$ & - & - \\
\hline 31 & & - & $\mathrm{Fr}$ & - & - \\
\hline 32 & & & - & - & - \\
\hline 33 & - & $\mathrm{Fr}$ & - & - & - \\
\hline 34 & & & $\mathrm{Fr}$ & - & - \\
\hline 35 & - & $\mathrm{Fr}$ & - & - & - \\
\hline 36 & & - & - & - & - \\
\hline
\end{tabular}

no cerrado em Pirassununga, (SP) que $C$. langsdorffii floresce logo após a queda de folhas concomitantemente com o brotamento. Isto parece indicar que a estratégia fenológica desta espécie pode variar em função da formação vegetal em que ocorre ou de fatores ambientais locais.

O padrão de desfolhamento apresentado por C. langsdorffii caracterizou-a como uma espécie semidecídua. Este mesmo padrão já foi observado para outras espécies do gênero, como C. chodotiana em uma floresta seca da Bolívia (Justiniano \& Fredericksen 2000) e $C$. pubiflora em uma savana da Venezuela (Ramírez 1978). Na população de C. langsdorffii da RSG, a queda de folhas ocorreu de forma regular e sincronizada e foi mais fortemente correlacionada com a diminuição da pluviosidade, sugerindo este fator como possível desencadeador da queda foliar apresentada por C. langsdorffii. Morellato (1991) estudando a fenologia de árvores na RSG, observou que a maioria das espécies perdem folhas no final da estação seca, um padrão que também ocorre próximo a Campinas numa floresta de altitude em Jundiaí (SP) (Morellato et al. 1989). Em outras regiões tropicais, como na Costa Rica (Frankie et al. 1974, Fournier 1976), México (WilliansLinera 1997) e Bolívia (Justiniano \& Fredericksen 2000), o pico de queda de folhas para a comunidade também tem sido observado nos meses mais secos do ano. De acordo com Matthes (1980), em seu estudo no Bosque dos Jequitibás, em Campinas (SP), a baixa umidade na estação seca e a diminuição da temperatura e do comprimento do dia estimulam a queda de folhas para a maioria das espécies. Martins (1982), estudando uma floresta semidecídua em Santa Rita do Passa Quatro (SP), sugeriu que fatores hídricos e térmicos regulam a estratégia foliar de diferentes espécies. Na RSG, fatores hídricos devem ser estímulos indutores mais importantes para a queda de folhas de Copaifera langsdorffii do que a diminuição da temperatura e do fotoperíodo durante a estação seca. Esta hipótese é reforçada pelo fato de mais de $50 \%$ dos indivíduos terem perdido folhas após a repentina diminuição na precipitação ocorrida durante a estação chuvosa, em fevereiro de 1992. Entre os indivíduos que não perderam folhas neste período, vários estavam estabelecidos em áreas de baixadas ou próximos a locais mais úmidos (mata de brejo) onde provavelmente existe disponibilidade permanente de água e ausência de estresse hídrico. Borchert (1999) observou que as mudanças foliares são progressivamente mais independentes da sazonalidade climática à medida em que as árvores crescem em microambientes com maior suprimento de água no solo, o qual protegeria as plantas contra o estresse hídrico sazonal. LemosFilho \& Mendonça-Filho (2000) investigando as relações entre estresse hídrico e eventos fenológicos numa floresta em Caratinga, MG, verificaram que para três espécies de leguminosas arbóreas estudadas a queda de folhas foi maior em locais mais secos da floresta. Em 1991, embora tenha ocorrido uma pequena diminuição na precipitação total em fevereiro, nenhum 
indivíduo observado perdeu folhas, sugerindo que a redução da pluviosidade durante a estação chuvosa, neste ano, não foi suficiente para estimular a abcisão foliar, o que corrobora a hipótese do estresse hídrico como fator indutor da queda de folhas para C. langsdorffii na RSG.

O brotamento de C. langsdorffii foi o evento fenológico mais regular e com maior sincronia entre os indivíduos da população. Ocorreu nos meses de setembro e outubro, que correspondem ao final da estação seca e início da estação chuvosa. Este período do ano coincide com o pico de espécies em brotamento na RSG (Morellato 1991), bem como em outras florestas tropicais (Frankie et al. 1974, Fournier 1976, Morellato et al. 1989). O fluxo sazonal de folhas novas é um traço característico das florestas tropicais sazonais (Longman \& Jenik 1987) sendo que a produção de folhas é promovida quando há aumento na disponibilidade de água e luz (Lieberman \& Lieberman 1984, Wright 1991, Aide 1993, Borchert 1994, Reich 1995) e esta tem sido considerada a fenofase mais marcante nas florestas semidecíduas do sudeste do Brasil (Morellato et al. 1989). Myers et al. (1998), por meio de um experimento de irrigação na Austrália, demonstraram que algumas espécies podem apresentar comportamento fenológico variável em função da umidade do solo, quantidade de água no caule e fatores climáticos como déficit de pressão de vapor. Morellato (1991) considerou que a precipitação após período de estresse hídrico foi o principal fator de indução do brotamento para a maioria das espécies na RSG. Provavelmente este fator também deve estimular o brotamento em Copaifera langsdorffii e a disponibilidade variável de água nos diferentes microambientes pode representar um fator adicional que explicaria as variações individuais observadas para as fenofases queda de folhas e brotamento.

$\mathrm{O}$ sincronismo do brotamento em um determinado período tem sido sugerido como uma estratégia para evitar a emissão de folhas novas durante estações desfavoráveis (van Schaik et al. 1993). Além disso, a produção sincronizada de folhas afeta a comunidade de herbívoros provocando flutuações em suas populações, pois a maioria dos recursos alimentares para estes consumidores ficam concentrados em poucos meses (Aide 1993, Angulo-Sandoval \& Aide 2000, Peters et al. 2001). Folhas produzidas simultaneamente devem sofrer menos danos do que folhas produzidas fora do pico de brotamento, já que quando há um aumento na disponibilidade de alimento os herbívoros são saciados mais facilmente (Janzen 1975). Dessa forma, a produção sincrônica de folhas, como a observada em Copaifera langsdorffii, pode favorecer o escape da herbivoria.

O padrão de florescimento apresentado por Copaifera langsdorffii pode ser considerado, segundo a classificação de Newstrom et al. (1994) como regular, pois a floração sempre ocorreu no mesmo período do ano (estação chuvosa) e supra-anual, porque ocorreu somente em alguns anos. Dias \& Oliveira-Filho (1996) também relataram um comportamento supra-anual para esta espécie em uma floresta semidecídua em Lavras (MG). A ausência de correlação entre variáveis climáticas (precipitação e temperatura) e o florescimento parece contraditória com a tendência de florescer na estação chuvosa. Dias \& Oliveira-Filho (1996) também observaram C. langsdorffii florescendo no período chuvoso e muitas espécies arbóreas do sudeste do Brasil florescem na mesma época (Morellato et al. 1989, 2000, Morellato 1991, SilberbauerGottsberger 2001). Algumas características favoráveis para o florescimento durante a estação chuvosa seriam: maiores temperaturas e índices pluviométricos acarretando aumento na decomposição da serapilheira e nos teores de nutrientes disponíveis para as plantas (Morellato 1992), variação na irradiação servindo como um fator próximo para iniciar e sincronizar o florescimento (Wright \& van Schaik 1994, Adler \& Kielpinski 2000) e aumento nas populações e atividade dos animais polinizadores durante este período (Wikander 1984).

Flores de curta duração como as de $C$. langsdorffii são comuns em espécies de plantas neotropicais (Cunninghan 1994). A duração da flor é um balanço entre o tempo em que ela fica exposta aos polinizadores, o custo de sua produção e manutenção para planta e o risco de ser parasitada. Flores de curta duração devem ocorrer em plantas onde é alta a probabilidade das flores serem visitadas no mesmo dia e ficarem rapidamente saturadas com pólen suficiente para produzir muitas sementes (Primack 1985b). O fato de Copaifera langsdorffii ser polinizada por abelhas (Apis melifera $\mathrm{e}$ Trigona sp.) que fazem visitas em grandes grupos (Crestana \& Kageyama 1989) reforça a relação da curta duração das flores e a alta probabilidade de serem polinizadas no mesmo dia. O florescimento sincronizado favorece a atração de polinizadores (Marquis 1988) e deve ser importante para a ocorrência de polinização cruzada entre indivíduos de $C$. langsdorffii na RSG.

A freqüência anual irregular de frutificação observada em Copaifera langsdorffii, durante o período de estudo e em anos anteriores, indicou que, na RSG, esta espécie apresenta um padrão de frutificação supra- 
anual (sensu Newstrom et al. 1994), com anos de intensa produção de frutos, seguidos por anos de pouca ou nenhuma produção. Leite \& Salomão (1992) citam a existência de um padrão bi ou trienal na produção de sementes de $C$. langsdorffii no Distrito Federal. Santos (1991) refere-se à ocorrência de padrões supra-anuais de frutificação para esta espécie em áreas de cerrado do Estado de São Paulo, o que também foi observado por Dias \& Oliveira-Filho (1996) em uma floresta semidecídua em Lavras (MG). Este padrão parece ser comum para o gênero Copaifera. Segundo Alencar (1984), C. multijuga apresenta frutificação bienal na Amazônia. Ramírez (1978) também verificou, para $C$. pubiflora, a ocorrência de períodos de frutificação massiva, seguidos de anos de pouca ou ausência de frutificação na Venezuela. A freqüência irregular de frutificação é considerada uma estratégia adaptativa para reduzir a predação de sementes através da saciação dos predadores (Janzen 1975, Tapper 1992). Períodos de grande produção de sementes seguidos de períodos de escassa produção regulam as populações de predadores (Silvertown 1980).

Copaifera langsdorffii foi observada com frutos maduros no final da estação seca. Morellato \& LeitãoFilho (1992) encontraram na Serra do Japi (SP) que espécies com frutos secos zoocóricos apresentaram frutos maduros na estação seca. Gautier-Hion (1990) também observou, em uma floresta da África, que frutos deiscentes tendiam a amadurecer no final da estação seca, quando as condições atmosféricas poderiam favorecer a deiscência da parede do fruto. Embora isto pareça verdadeiro para C. langsdorffii na RSG, esta fenofase não apresentou correlação significativa com parâmetros climáticos analisados. De qualquer forma, a maturação dos frutos antes da estação chuvosa proporcionaria às sementes máxima chance de germinação devido aos teores favoráveis de umidade (Frankie et al. 1974, van Schaik et al. 1993). Além disso, as plântulas poderiam desenvolver o sistema radicular durante a estação de chuvas, antes de serem submetidas ao estresse hídrico da estação seca subseqüente (Janzen 1967, Fournier 1976).

Outro fator que influencia a produção, o valor nutritivo e a apresentação temporal dos frutos é a pressão seletiva de dispersores e predadores de sementes (Krebs 1994). A duração da frutificação de Copaifera langsdorffii pode ser classificada como intermediária ( $>1 \mathrm{e}<5$ meses, sensu Newstrom et al. 1994). Este é um padrão contrastante comparado com o apresentado por outras Leguminosae. Felfili et al. (1999) observaram, num cerrado do Brasil Central, que
Stryphnodendron adstringens apresenta frutos em diversos estádios de maturação durante o ano todo. Espécies que apresentam frutificação relativamente curta com episódios de frutificação sincronizados entre indivíduos coespecíficos, como apresentado por $C$. langsdorffii neste estudo, tendem a apresentar forte sazonalidade na frutificação (Sun et al. 1996).

Na RSG, Copaifera langsdorffii é uma planta com alta fecundidade, pois produz grande quantidade de frutos, num curto período de tempo. Os frutos apresentam baixo teor nutritivo individual, já que o arilo é composto principalmente por carboidratos (Rodrigues et al. 2000). Esse conjunto de características é típico da "síndrome da superabundância" (sensu McKey 1975). Plantas que apresentam estas características tendem a atrair uma ampla variedade de animais com dieta generalista para realizar a dispersão das sementes. Na RSG, Copaifera langsdorffii produz frutos maduros fora do pico de frutificação de espécies zoocóricas (Morellato 1991) e, embora tenha frutos tipicamente ornitocóricos, frutificar neste período favorece a atração de outros animais dispersores além das aves (Pedroni 1995). Segundo Janzen (1975), os padrões de frutificação de espécies zoocóricas, por dependerem de animais para a disseminação de seus diásporos, estão sob forte pressão seletiva dos seus dispersores. Na RSG, Morellato (1991) observou que as espécies que possuem frutos ornitocóricos (incluindo $C$. langsdorffii) frutificam de forma seqüencial ao longo do ano. Estas observações concordam com a hipótese de que espécies que utilizam o mesmo grupo de dispersores teriam vantagens no deslocamento dos períodos de frutificação para minimizar a competição por dispersores (Snow 1965, Gautier-Hion 1990).

$\mathrm{Na}$ RSG, o estabelecimento e a reprodução de Copaifera langsdorffii parecem ser favorecidos pelo ritmo fenológico que ela apresenta. Na estação chuvosa, as árvores maximizam o ganho de carbono por estarem com as copas plenamente desenvolvidas, o que influencia o abundante florescimento e a alta produtividade de frutos. Na estação seca, as plantas perdem folhas aliviando o déficit hídrico e abrem os frutos quando a umidade do ar é favorável à deiscência, expondo as sementes ariladas aos dispersores. A estratégia vegetativa parece ser mais influenciada por fatores climáticos (fatores próximos) enquanto a estratégia reprodutiva, pelas interações entre planta e animal (fatores finais). A irregularidade, individual e populacional, da frutificação pode representar uma estratégia para evitar a ação de predadores e garantir a dispersão de sementes. 
Agradecimentos - Ao Conselho Nacional de Pesquisa (CNPq) e Fundação de Amparo a Pesquisa do Estado de São Paulo (FAPESP) pela bolsa concedida ao primeiro autor e à Profa. Dra. L. Patrícia C. Morellato por permitir o uso de dados não publicados. À Fundação José Pedro de Oliveira por permitir o acesso a Reserva Municipal de Santa Genebra.

\section{Referências bibliográficas}

ADLER, G.H. \& KIELPINSKI, K.A. 2000. Reproductive phenology of a tropical canopy tree, Spondias mombim. Biotropica 32:686-692.

AIDE, M. 1993. Synchronous leaf production and herbivory in juveniles of Gustavia superba. Oecologia 88:511-514.

ALENCAR, J.C. 1984. Estudos silviculturais de uma população natural de Copaifera multijuga Hayne Leguminosae, na Amazônia Central. 3. Distribuição espacial da regeneração natural pré-existente. Acta Amazonica 14:255-279.

ALMEIDA, E.M. \& ALVES, M.A. 2000. Fenologia de Psychotria nuda e P. brasiliensis (Rubiaceae) em uma área de Floresta Atlântica no Sudeste do Brasil. Acta Botanica Brasilica 14:335-346.

ANGULO-SANDOVAL, P. \& AIDE, M. 2000. Leaf phenology and leaf damage of saplings in the Luquilo Experimental Forest, Puerto Rico. Biotropica 32:415-422.

AUGSPURGER, C.K. 1983. Phenology, flowering synchrony and fruit set of six neotropical shrubs. Biotropica 15:257-267.

AUGSPURGER, C.K. 1996. A cue for synchronous flowering. In The ecology of a tropical rain forest: seasonal rhythms and long-term changes. (E.G. Liegh Jr., A.S. Rand \& D.M. Windsor, eds.). Smithsonian Institution Press, Washington, p.133-150.

BATALHA, M.A. \& MANTOVANI, W. 2001. Reproductive phenological patterns of cerrado plant species at the Pé de Gigante Reserve (Santa Rita do Passa Quatro, SP, Brazil): a comparison between the herbaceous and woody floras. Revista Brasileira de Biologia 60:129-145.

BATALHA, M.A., ARAGAKI, S. \& MANTOVANI, W. 1997. Variações fenológicas das espécies do cerrado de Emas - Pirassununga, SP. Acta Botanica Brasilica 11:61-78.

BORCHERT, R. 1980. Phenology and ecology of a tropical tree Erythryna poeppigiana O. F. Cook. Ecology 61:1065-1074.

BORCHERT, R. 1994. Soil and stem water storage determine phenology and distribution of tropical dry forest trees. Ecology 75:1437-1449.

BORCHERT, R. 1999. Climate periodicity, phenology, and cambium activity in tropical dry forest trees. Iawa Journal 20:239-247.
CRESTANA, C.M. \& KAGEYAMA, P.Y. 1989. Ecologia de polinização de Copaifera langsdorffii Desf. (Leguminosae - Caesalpinioideae), "o óleo de copaíba". Revista do Instituto Florestal 1:201-214.

CUNNINGHAN, S.A. 1994. Measuring the relationship between floral duration and fruit set for Hamelia patens (Rubiaceae). Biotropica 26:227-229

DIAS, H.C.T. \& OLIVEIRA-FILHO, A.T. 1996. Fenologia de quatro espécies arbóreas de uma floresta estacional semidecídua em Lavras, MG. Cerne 2:66-88

FELFILI, J.M., SILVA JUNIOR, M.C., DIAS, B.J. \& REZENDE, A.V. 1999. Estudo fenológico de Stryphnodendrom adstringens (Mart.) Coville no cerrado sensu stricto da Fazenda Água Limpa no Distrito Federal, Brasil. Revista Brasileira de Botânica 22:83-90.

FLOWERDEW, J.R. \& GARDNER, G. 1978. Small rodent population and food supply in a Derbyshire ashwood. Journal of Animal Ecology 47:725-740.

FOURNIER, L.A. 1976. Observaciones fenologicas en el bosque humedo premontano de San Pedro de Montes Oca, Costa Rica. Turrialba 26:54-59.

FRANKIE, G.W., BAKER, H. \& OPLER, P.A. 1974. Comparative phenological studies of trees in tropical wet and dry forests in the lowlands of Costa Rica. Journal of Ecology 62:81-919.

GAUTIER-HION, A. 1990. Interactions among fruit and vertebrate fruit-eaters in an African tropical rain forest. In Reproductive ecology of tropical forest plants. (K.S. Bawa \& M. Hadley, eds.). Man and the Biosphere Series. The Parthenon Publishing Group, Paris, v.7, p.219-230.

HARRISON, R.D., YAMAMURA, N. \& INOUE, T. 2000. Phenology of a road commom fig in Sarawak. Ecological Research 15:47-61.

JANZEN, D. 1967. Synchronization of sexual reproduction of trees within the dry season in Central America. Evolution 21:620-637.

JANZEN, D. 1971. Seed predation by animals. Annual Review of Ecology and Systematics 2:465-492

JANZEN, D. 1975. Behaviour of Hymenaea courbaril when its predispersal seed predator is absent. Science 189:145-147.

JUSTINIANO, M.J. \& FREDERICKSEN, T.S. 2000. Phenology of tree species in Bolivian dry forests. Biotropica 32:276-281.

KÖPPEN, W. 1948. Climatologia, Fondo de Cultura Económica, México.

KREBS, C.J. 1994. Ecology: the experimental analysis of distribution and abundance. Harper Collins, New York.

LEITÃO FILHO, H.F. 1995. A vegetação da Reserva de Santa Genebra. In Ecologia e preservação de uma floresta tropical urbana: Reserva de Santa Genebra. (H.F. Leitão Filho \& L.P.C. Morellato, eds.). Universidade Estadual de Campinas, Campinas, p.19-29.

LEITE, A.M.C. \& SALOMÃO, A.N. 1992. Estrutura populacional de regenerantes de copaíba (Copaifera langsdorffii Desf.) em mata ciliar do Distrito Federal. Acta Botanica Brasilica 6:123-134. 
LEMOS-FILHO, J.P. \& MENDONÇA-FILHO, C.V. 2000. Seasonal changes in the water status of three woody legumes from the Atlantic forest, Caratinga, Brazil. Journal of Tropical Ecology 16:21-32.

LIEBERMAN, D. \& LIEBERMAN, M. 1984. The causes and consequences of synchronous flushing in a dry tropical forest. Biotropica 16:193-201.

LONGMAN, K.A. \& JENIK, J. 1987. Tropical forest and its enviroments. Longman Scientific \& Technical, New York.

MACHADO, I.C.S., BARROS, L.M. \& SAMPAIO, E.V.S.B. 1997. Phenology of Caatinga species at Serra Talhada, PE, Northeastern Brazil. Biotropica 29:57-68.

MARQUIS, R.J. 1988. Phenological variation in the Neotropical understory shrub Piper arieianum: causes and consequences. Ecology 69:1552-1565.

MARTINS, F.R. 1982. O balanço hídrico seqüencial e o caráter semidecíduo de floresta do Parque Estadual de Vassununga, Santa Rita do Passa Quatro, SP. Revista Brasileira de Estatística 43:353-391.

MATTHES, L.A.F. 1980. Composição florística, estrutura e fenologia de uma floresta residual do planalto paulista: Bosque dos Jequitibás. Dissertação de mestrado, Universidade Estadual de Campinas, Campinas.

MCKEY, D. 1975. The ecology of coevolved seed dispersal systems. In Coevolution of animals and plants. (L.E. Gilbert \& P.H. Raven, eds.). University of Texas, Austin, p.159-191.

MIRANDA, I.S. 1995. Fenologia do estrato arbóreo de uma comunidade de cerrado em Alter-do-Chão, PA. Revista Brasileira de Botânica 18:235-241.

MORELLATO, L.P.C. 1991. Estudo da fenologia de árvores, arbustos e lianas de uma floresta semidecídua no sudeste do Brasil. Tese de doutorado, Universidade Estadual de Campinas, Campinas.

MORELLATO, L.P.C. 1992. Sazonalidade e dinâmica de ecossistemas florestais na Serra do Japi. In História natural da Serra do Japi: ecologia e preservação de uma área florestal no Sudeste do Brasil (L.P.C. Morellato, ed.). Universidade Estadual de Campinas/Fundação de Amparo à Pesquisa do Estado de São Paulo, Campinas, p.98-110.

MORELLATO, L.P.C. \& LEITÃO FILHO, H.F. 1992. Padrões de frutificação e dispersão de sementes na Serra do Japi. In História natural da Serra do Japi: ecologia e preservação de uma área florestal no Sudeste do Brasil (L.P.C. Morellato, ed.). Universidade Estadual de Campinas/Fundação de Amparo à Pesquisa do Estado de São Paulo, Campinas, p.112-140.

MORELLATO, L.P.C., RODRIGUES, R.R., LEITÃO FILHO, H.F. \& JOLY, C.A. 1989. Estudo comparativo da fenologia de espécies arbóreas de floresta de altitude e floresta mesófila semidecídua na Serra do Japi, Jundiaí, São Paulo. Revista Brasileira de Botânica 12:85-98.
MORELLATO, L.P.C., TALORA, D.C., TAKAHASI, A., BENKLE, C.C., ROMERA, E.C. \& ZIPPARRO, W.B. 2000. Phenology of Atlantic Rain Forest trees: a comparative study. Biotropica 32:811-823.

MYERS, B.A., WILLIANS, R.J., FORDYCE, I., DUFF, G.A. \& EAMUS, D. 1998. Does irrigation affect leaf phenology in deciduous and evergreen trees of the savannas of northern Australia? Australian Journal of Ecology 23:329-339.

NEWSTROM, L.E., FRANKIE, G.W. \& BAKER, H.G. 1994. A new classification for plant phenology based on flowering patterns in lowland tropical forest trees at La Selva, Costa Rica. Biotropica 26:141-159.

OLIVEIRA, J.B. 1980. Características morfológicas, analíticas, limitações e aptidão agrícola dos solos da quadrícula de Campinas. Circular Técnica n. 109, Instituto Agronômico de Campinas, Campinas.

OPLER, P.A., FRANKIE, G.W. \& BAKER, H.G. 1976. Rain fall as a factor in the release, timing and synchronization of anthesis by tropical trees and shrubs. Journal of Biogeography 3:231-236.

PEDRONI, F. 1995. Ecologia da copaíba. In Ecologia e preservação de uma floresta tropical urbana: Reserva de Santa Genebra (H.F Leitão Filho \& L.P.C. Morellato, eds.). Universidade Estadual de Campinas, Campinas, p.70-76.

PETERS, P.J., READ, J. \& SANSON, G.D. 2001. Variation in the guild composition of herbivorous insect assemblages among co-occuring plant species. Austral Ecology 26:385-399.

PRIMACK, R.B. 1985a. Patterns of flowering phenology in communities, populations, individuals, and single flowers. In The population structure of vegetation (J. White, ed.). Handbook of vegetation science, Dr. W. Junk Publ., Dordrecht, v.3, p.571-593.

PRIMACK, R.B. 1985b. Longevity os individuals flowers. Annual Review of Ecology and Systematics 16:15-37.

RAMÍREZ, N. 1978. Dinamica demografica, depredacion de semillas y mecanismos de dispersion en Copaifera pubiflora Bentham (Leguminosae: Caesalpinioideae). Trabajo de Graduación, Universidad Central de Venezuela, Caracas.

REICH, P.B. 1995. Phenology of tropical forests: patterns, causes and consequences. Canadian Journal of Botany 73:164-174.

REICH, P.B. \& BORCHERT, R. 1984. Water stress and tree phenology in a tropical dry forest in the lowlands of Costa Rica. Journal of Ecology 72:61-74.

RIVERA, G. \& BORCHERT, R. 2001. Induction of flowering in tropical trees by a 30-min reduction in photoperiod: evidence from field observations and herbarium specimens. Tree Physiology 21:201-212.

RODRIGUES, M., BELFORT, H., CAMPOLINA, C. \& GARCIA, Q. 2000. O tucanuçu (Ramphastos toco) como agente dispersor de sementes de copaíba. Melopsittacus 3:6-11. 
SANTOS, F.A.M. 1991. Padrão espacial de jovens em relação a adultos de espécies arbóreas de cerrado que ocorrem no estado de São Paulo. Tese de doutorado, Universidade Estadual de Campinas, Campinas.

SILBERBAUER-GOTTSBERGER, I. 2001. A hectare of cerrado. II. Flowering and fruiting of thick-stemmed woody species. Phyton-Annales Rei Botanicae 41:129-158.

SILVERTOWN, J.W. 1980. The evolutionary ecology of mast seeding in trees. Biological Journal of the Linnean Society 14:235-250.

SMYTHE, N. 1970. Relationships between fruiting seasons and seed dispersal methods in a neotropical forest. American Naturalist 104:25-35.

SNOW, D.W. 1965. A possible selective factor in the evolution of fruiting seasons in tropical forest. Oikos $15: 274-281$.

SUN, C., KAPLIN, B.A., KRISTENSEN, K.A., MUNYALIGOGA, V., MVUKIYUMWAMI, J., KAJONDO, K. \& MOERMOND, T.C. 1996. Tree phenology in a tropical montane forest in Rwanda. Biotropica 28:668-681.

TAPPER, P.G. 1992. Irregular fruiting in Fraxinus excelsior. Journal of Vegetation Science 3:41-46.

VAN SCHAIK, C.P., TERBORGH, J.W. \& WRIGHT, S.J. 1993. The phenology of tropical forest: adaptative significance and consequences of consumers. Annual Review of Ecology and Systematics 24:353-377.
VELOSO, H.P., RANGEL FILHO, A.L.R. \& LIMA, J.C.A. 1991. Classificação da vegetação brasileira, adaptada a um sistema universal. IBGE, Rio de Janeiro.

WIKANDER, T. 1984. Mecanismos de dispersión de diasporas de una selva decidua en Venezuela. Biotropica 16:276283.

WILLIANSLINERA, G. 1997. Phenology of deciduous and broad-leaved evergreen tree species in a Mexican tropical lower montane forest. Global Ecology and Biogeography Letters 6:115-127.

WILLIAMS, R.J., MYERS, B.A., EAMUS, D. \& DUFF, G.A. 1999. Reproductive phenology of woody species in a North Australian Tropical savanna. Biotropica 31:626-636.

WRIGHT, S.J. 1991. Seasonal drought and the phenology of understory shrubs in a tropical moist forest. Ecology 72:1643-1657.

WRIGHT, S.J. \& van SCHAIK, C.P. 1994. Light and the phenology of tropical trees. American Naturalist 143:192-199.

ZAGT, R.J. 1997. Pre-dispersal and early post-dispersal demography, and reproductive litter production in the tropical tree Dicymbe altsonii in Guyana. Journal of Tropical Ecology 13:511-526.

ZAR, J.H. 1999. Biostatiscal analysis. Prentice-Hall, New Jersey. 